\title{
The altitude profile of the cosmic ray atmospheric cut-off
}

\author{
Stepan Poluianov ${ }^{a, *}$ and Alexander Mishev ${ }^{a}$ \\ a University of Oulu, \\ 90014 Oulu, Finland \\ E-mail: stepan.poluianov@oulu.fi, alexander.mishev@oulu.fi
}

Neutron monitors are the main ground-based instruments for continuous measurements the cosmicray intensity operating over more than five decades. Those instruments are energy-integrating detectors with count rates governed by the atmospheric and geomagnetic cutoffs. The geomagnetic cutoff dominates (up to $17 \mathrm{GV}$ in rigidity) over most of the globe. However, it is negligible in the polar regions, and there, the atmospheric cutoff is important. The atmospheric cutoff depends on the elevation of the instrument above sea level (on the atmospheric depth), and it is estimated as $1 \mathrm{GV}$ for cosmic-ray protons at sea level. However, the atmospheric cutoff is not precisely known at higher altitudes. This is specifically important for studies based on high-altitude polar neutron monitors, which count rate is solely defined by the atmospheric cutoff.

We present a newly estimated altitude profile of the atmospheric cutoff for cosmic-ray protons, which can be used in analysis of both galactic cosmic rays and solar energetic particles. We computed the profile using two methods. The first one is based on Monte Carlo simulation of the cosmic-ray induced cascade in the atmosphere with the PLANETOCOSMICS code. The second one uses recently computed and verified neutron monitor yield function by Mishev et al., 2020, which explicitly considers the efficiency of the instrument. Both methods agree reasonably well, though the yield-function based one provides a more conservative result, as expected. There are two definitions of solar-particle sub-GLE (sub-Ground-Level-Enhancement) events by Raukunen et al. (2018) and Poluianov et al. (2018) based on different principles. Considering the derived in this study atmospheric cutoff at altitudes about $3000 \mathrm{~m}$ a.s.l., we conclude: there is no contradiction between the definitions.

$3^{\text {th }}$ International Cosmic Ray Conference (ICRC 2021)

July 12 th - 23rd, 2021

Online - Berlin, Germany

\footnotetext{
${ }^{*}$ Presenter
} 


\section{Introduction}

Relativistic cosmic rays constantly bombard the Earth and can produce multiple secondary particles in atmospheric cascades. The global network of ground-based neutron monitors (NM) exists since the 1950. Those instruments are designed to count the cosmic-ray cascades by detection of their secondary hadronic component (mainly neutrons).

NM location is characterised with the geomagnetic and atmospheric cut-offs. Those cut-offs are the minimum rigidity/energy of primary cosmic-ray particles necessary to have them registered by an NM in that location. The geomagnetic cut-off is caused by deflection of charged cosmic rays by the magnetic field of the Earth. Its cut-off rigidity is highest in the equator region and almost null near the geomagnetic poles. The atmospheric cut-off is caused by attenuation of the cosmic ray cascade in the deep layers of the atmosphere. Therefore, the cut-off energy is related to the thickness of the atmosphere above a given location (altitude). The maximum atmospheric cut-off is at sea level, where the atmosphere is $1033 \mathrm{~g} / \mathrm{cm}^{2}$ thick. The sea-level atmospheric cut-off energy is known ( $\sim 1 \mathrm{GV}$ in rigidity or $\sim 433 \mathrm{MeV}$ in energy for cosmic-ray protons) from cosmic-ray latitude surveys. The atmospheric cut-off decreases with decrasing atmospheric depth (and with growing altitude), but exact values were poorly known so far.

The altitude profile of the atmospheric cut-off is important for analysis of so-called groundlevel enhancement (GLE) and sub-GLE events [1,2] caused by solar energetic particles (SEP). In particular, the atmospheric cut-off is dominating in the polar regions, where the geomagnetic cut-off is negligible. There are several high-altitude NMs among polar stations, which have greatly reduced atmospheric cut-off (and almost null geomagnetic cut-off): SOPO/SOPB (elevation $2820 \mathrm{~m}$ a.s.l., the atm. depth $695 \mathrm{~g} / \mathrm{cm}^{2}$ ), DOMC/DOMB (3233 m a.s.1., $654 \mathrm{~g} / \mathrm{cm}^{2}$ ). Their exeptional sensitivity to low-energy cosmic rays, thanks to the low atmospheric cut-off, caused an introduction of the class of sub-GLE events. There are two definitions of sub-GLEs [1,2]: the first is observational, the second is based on the energy threshold. Unfortunately, since the atmospheric cut-off energies were unknown for the high-altitude polar NMs, it was unclear how the definitions are related to each other. Here, we present the quantitatively estimated atmospheric cut-off energies for cosmic rays registered by NMs at different altitudes and clarify the sub-GLE defintions.

\section{Physical approach}

Within the physical approach, we simulated the development of the cosmic-ray cascade in the atmosphere with a Monte Carlo code. We used the Geant4-based code PLANETOCOSMICS [3, 4] specifically designed for that purpose, which shows sufficiently good (for our purpose) agreement with experimental data. We simulated a full-size spherical atmosphere with composition and properties according to NRLMSISE-00 [5]. We did not consider the geomagnetic field because we assumed that the simulation should be done with the null geomagnetic cut-off. Since the main focus of this work is at solar energetic particles, cosmic rays were represented as monoenergetic fluxes of primary protons shot with two angular distributions: isotropic and vertical. These are two extreme cases bounding realistic SEP events that have some degree of angular anisotropy. The energies of primary particles were set within $200-500 \mathrm{MeV}$. The simulated cascade was recorded as the intensity of secondary particles at selected atmospheric depths (altitudes) from 500 to 1033 

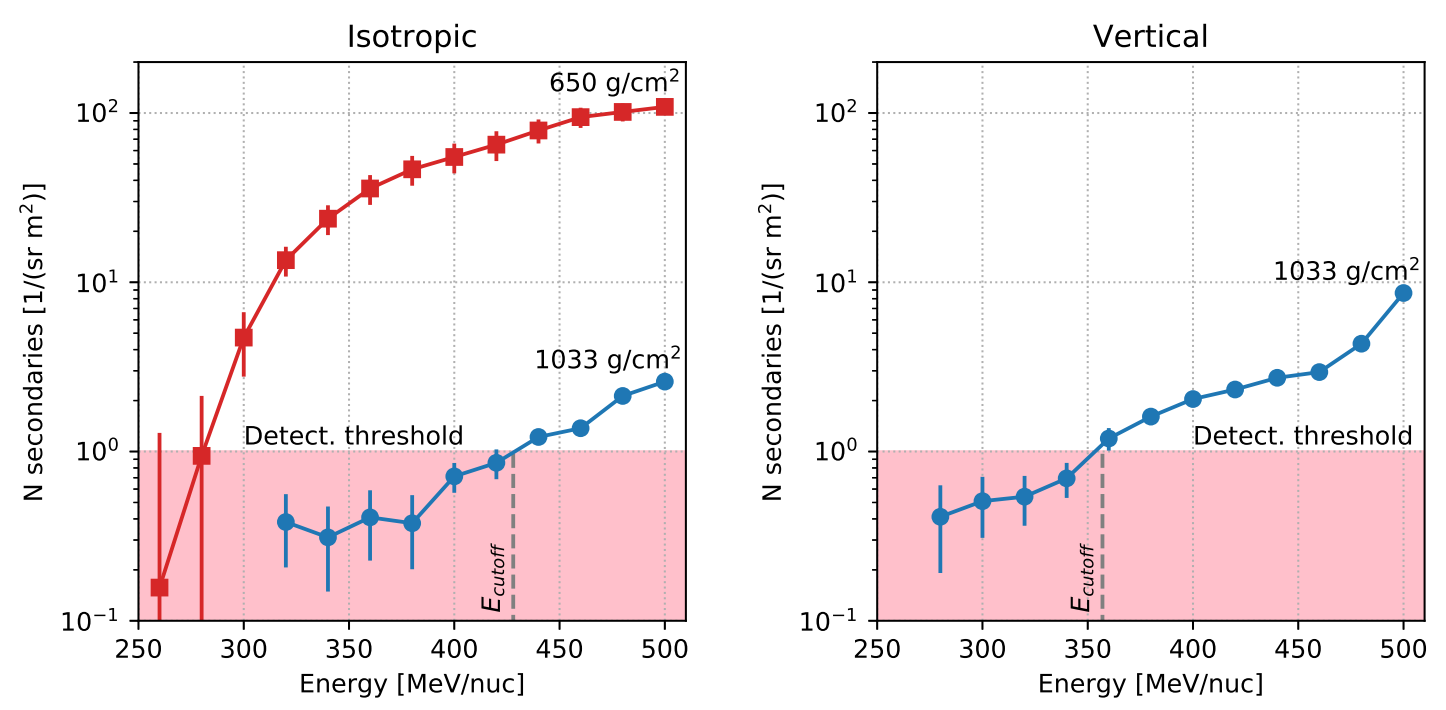

Figure 1: Fluxes of secondary particles (neutrons) simulated using primary protons with isotropic (left panel) and vertical (right panel) incidence. The energy at X-axes is the kinetic energy of primary particles. The pink area indicates the detection threshold, above which particles are considered as detectable.

$\mathrm{g} / \mathrm{cm}^{2}$ (from about $5 \mathrm{~km}$ to $0 \mathrm{~km}$ above sea level). Since cosmic-ray cascades are most probably registered by ground-based neutron monitors via secondary neutrons, we focused at analysis of the neutrons in the atmosphere. The simulated intensity of secondary neutrons versus the energy of incident particles is shown in Figure 1. As expected, the number of secondaries produced in a cascade is directly dependent on the initial energy of the primary. Additionally, the cascade is weaker in deeper atmosphere (sea level, $1033 \mathrm{~g} / \mathrm{cm}^{2}$ ) than at more shallow depth $\left(650 \mathrm{~g} / \mathrm{cm}^{2}\right)$.

To estimate the atmospheric cut-off, we defined the detection criterion as one secondary neutron per unit intensity of the cosmic-ray incident (primary) protons bombarding the top of the modelled atmosphere. We assumed that below this value, the probability of registration is low. This threshold is indicated with pink area in Figure 1. The energy, at which the simulated intensity of secondaries crosses the threshold, is the atmospheric cut-off energy for the given depth (altitude) within the physical approach. The estimated cut-off energy at sea level $\left(1033 \mathrm{~g} / \mathrm{cm}^{2}\right)$ for isotropic incident protons is $428 \pm 9 \mathrm{MeV}$. It is in good agreement with the experimental value of about $433 \mathrm{MeV}$ obtained in latitude surveys.

The atmospheric cut-off energies for the isotropic and vertical cases over the range of depths (altitudes) are shown in Figure 2 and Table 1. We can see there that the simulation with the vertical distribution (green line) provides systematically lower cut-off than the isotropic one (blue line). This is caused by some degree of cascade inclination that is always present in the more realistic isotropic case. Therefore, secondaries have less probability to reach deeper layers of the atmosphere, which causes higher cut-off energies. In contrast, vertically incident cascade traverse the minimum amount of air mass, which explains the lower cut-off. The isotropic case provides more conservative estimate of the atmospheric cut-off than the vertical one, and we rely on it within the physical approach. 


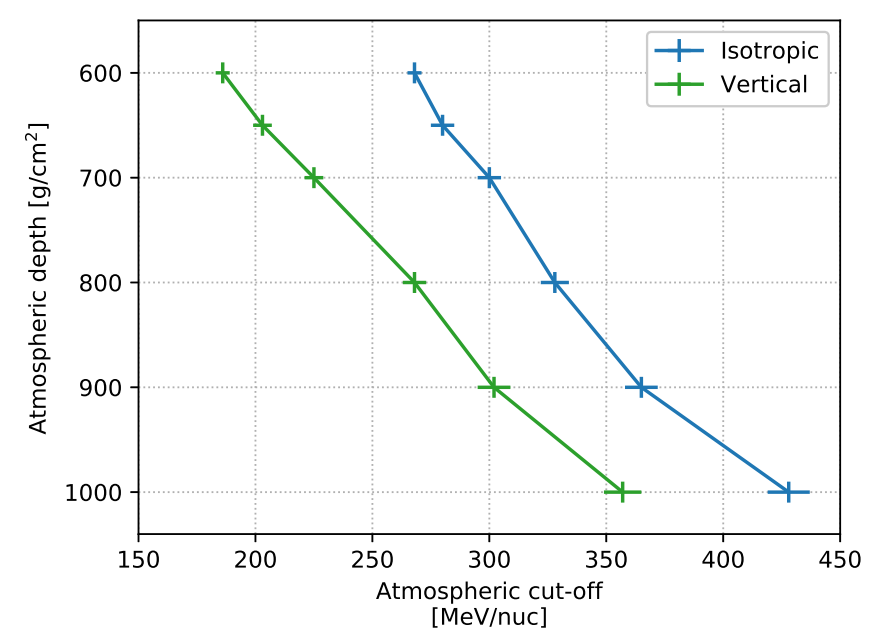

Figure 2: Atmospheric cut-off energy estimated by the physical approach for protons as a function of the depth. The values are calculated for the isotropical and vertical incident particles.

\section{Instrumental approach}

In the section above, it was assumed that the detector can register cascade neutrons with $100 \%$ efficiency, however in reality, the registration efficiency is not perfect. Therefore, we used another "instrumental" approach to take it into account. We considered the standard 6NM64 neutron monitor [6], which is in use at most of cosmic ray stations. The calculations below are based on the yield (response) function of the instrument [7, 8]. This function represents the response of the detector (in counts) on the unit flux of cosmic ray particles of given type with given energy. The yield function takes into account both the development of the cosmic-ray cascade in the atmosphere and response of the instrument including its registration efficiency and geometry.

In particular, we used the recent NM yield function by [9], which is computed for different altitudes in addition to sea level. The function is verified with direct space-borne measurements [10] and latitude surveys $[11,12]$. The NM count rate $N(h)$ can be calculated as:

$$
N(h)=\sum_{i=p, \alpha} \int_{P_{\text {gm. cut }}}^{\infty} Y_{i}(P, h) J_{i}(P) d P,
$$

where $h$ is the atmospheric depth $\left[\mathrm{g} / \mathrm{cm}^{2}\right], P$ is the magnetic rigidity of cosmic-ray particles [GV], $i$ is the type of primaries ( $\mathrm{p}$ - protons, $\alpha$ - alpha-particles, the latter ones effectively represent heavier ions [8]), $P_{\text {gm.cut }}$ is the local geomagnetic cut-off rigidity, $Y_{i}(P, h)$ is the NM yield function [counts $\left.\mathrm{sr} \mathrm{m}^{2}\right], J_{i}(P)$ is the differential flux cosmic-ray particles [1/(s sr $\left.\left.\mathrm{m}^{2} \mathrm{GV} / \mathrm{nuc}\right)\right]$.

The geomagnetic cut-off $P_{\text {gm.cut }} \approx 0 \mathrm{GV}$ in the polar regions, and the atmospheric cut-off is dominant there. Thus, the NM count rate caused by $i$-th type of cosmic-ray particles is

$$
N_{i}(h)=\int_{0}^{\infty} Y_{i}(E, h) J_{i}(E) d E,
$$

where $E$ is the kinetic energy [GeV/nuc] and henceforth $J_{i}(E)$ is in units [1/(s sr m$\left.\left.{ }^{2} \mathrm{GeV} / \mathrm{nuc}\right)\right]$. Note the rigidity-to-energy conversion of the integral. 
The yield function energy profile has no apparent cut-off, it asymptotically approaches zero [9]. Therefore, we needed to define the detection criterion to estimate the atmospheric cut-off. The count rate integral (Equation 2) can be represented as a sum of two components corresponding to the lower and higher parts of the energy spectrum split with some energy threshold $E_{c}$ :

$$
N_{i}(h)=\underbrace{\int_{E_{c}}^{\infty} Y_{i}(E, h) J_{i}(E) d E}_{I_{1, i}\left(h, E_{c}\right)}+\underbrace{\int_{0}^{E_{c}} Y_{i}(E, h) J_{i}(E) d E}_{I_{2, i}\left(h, E_{c}\right)} .
$$

The majority of NM counts are caused by integral $I_{1, i}\left(h, E_{c}\right)$, while $I_{2, i}\left(h, E_{c}\right)$ is a remainder due to low-energy $\left(E<E_{c}\right)$ particles. As mentioned above, we assumed the contribution of $\alpha$-particles and heavier species in SEP events being negligible to the NM count rate during GLEs or sub-GLEs. Therefore, we focused on cosmic-ray protons, and set $i=\mathrm{p}$ in the following computations.

We defined the detection threshold within the instrumental method as the maximum $E_{c}$, when contribution of the remainder integral $I_{2, p}\left(h, E_{c}\right)$ to the total count rate $N(h)$ is indistinguishable. This is possible because of the natural random variability of the count rate $N$, which has the Poisson probability distribution with the mean value $\langle N\rangle$ and standard deviation $\sigma=\sqrt{\langle N\rangle}$. We calculated the theoretical mean count rate $\langle N\rangle$ with the yield function $Y_{i}(E, h)[9]$ and the differential spectrum of galactic cosmic rays $J_{i}(E)$. For the latter, we used the standard force-field approximation [13] of the galactic cosmic ray particle flux described as:

$$
J_{i}(E)=J_{\mathrm{LIS}, i}\left(E+\frac{Z_{i}}{A_{i}} \phi\right) \cdot \frac{E\left(E+2 E_{0}\right)}{\left(E+\frac{Z_{i}}{A_{i}} \phi\right)\left(E+\frac{Z_{i}}{A_{i}} \phi+2 E_{0}\right)},
$$

where $J_{\mathrm{LIS}, i}$ is the differential flux of $i$-th particles in the local interstellar medium [1/(s sr $\mathrm{m}^{2}$ $\mathrm{GeV} / \mathrm{nuc})], Z$ and $A$ are the charge and mass numbers of $i$-th particles, $\phi$ is the heliospheric modulation potential [GV] reflecting solar activity, $E_{0}=0.938 \mathrm{GeV}$ is the rest mass of a proton. The spectrum $J_{\text {LIS }, i}$ was calculated with parametrisation of [14] and the fraction of $Z \geq 2$ particles as 0.353 [10]. We used three values of $\phi$ corresponding different levels of solar activity: $\phi=652$ MV (mean value for 1951-2019, http://cosmicrays.oulu.fi/phi/phi.html), 300 MV (low activity) and $1200 \mathrm{MV}$ (high activity).

Then, we calculated and normalized the reminder integral with the standard deviation as $I_{2, p}\left(h, E_{c}\right) / \sigma(h)$. The result as a function of energy $E_{c}$ and depth $h$ is shown in Figure 3. We defined a detection threshold, which is invariant to the total count rate $N(h)$ and depth $h$ :

$$
T=\frac{I_{2, p}^{\text {th }}(h)}{\sigma(h)}=\text { const. }
$$

Thus, the remainder integral $I_{2, p}\left(h, E_{c}\right) / \sigma(h)$ is considered as an indistinguishable contribution to the count rate, if its value is lower than $T$. To obtain the exact value of threshold $T$, we used the experimental data from latitude surveys [e.g., 15, 16], namely known atmospheric cut-off energy $E_{\text {atm.cut }} \approx 433 \mathrm{MeV}$ at sea level $\left(h=1033 \mathrm{~g} / \mathrm{cm}^{2}\right)$. After that, we interpolated the remainder integrals for different altitudes with the threshold (horizontal line in Figure 3) and calculated the atmospheric cut-off energies as energies $E_{c}$ at the intersections of $I_{2, p}\left(h, E_{c}\right) / \sigma(h)$ and $T$. The result $E_{\text {atm.cut }}$ of the instrumental method are presented in Figure 4 and Table 1 along with the 


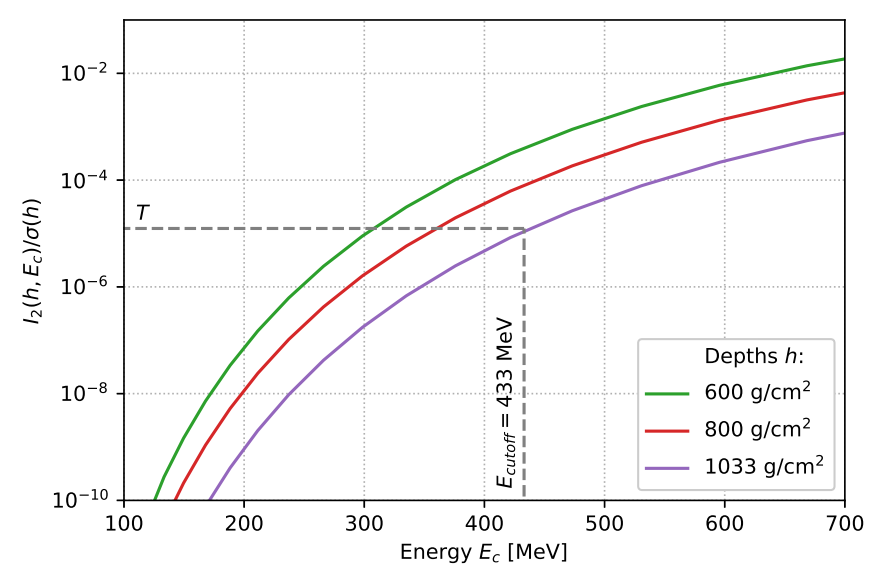

Figure 3: Remainder integral $I_{2, p}\left(h, E_{c}\right) / \sigma(h)$ (Equation 3) for different depths $h$ (see the legend). The dashed line indicates threshold $T$ (Equation 5) estimated from the known cut-off energy $E_{\text {atm.cut }} \approx 433 \mathrm{MeV}$ for the sea level $\left(1033 \mathrm{~g} / \mathrm{cm}^{2}\right)$. All plotted data correspond to calculations with $\phi=652 \mathrm{MV}$ (mean solar modulation for 1951-2019).

values of the physical approach. We repeated the cut-off calculations for different levels of solar activity $(\phi=652,300$ and $1200 \mathrm{MV})$ and found out that the solar variability causes a very minor effect $( \pm 1 \%)$. Therefore, we used the result corresponding to the mean solar activity of 1951-2019 $(\phi=652 \mathrm{MV})$ for discussions below.

\section{Discussion and conclusions}

Figure 4 presents the altitude profiles of the atmospheric cut-off energies calculated with the physical and instrumental methods. Both curves have a graduate increase of $E_{\text {atm.cut }}$ with growing depth $h$ towards sea level. The instrumental estimate shows greater values of the cut-off than the physical one. This is due to the limited detection efficiency of neutron monitors taken into account with the yield function in the instrumental method, while $100 \%$ registration efficiency was assumed in the physical method. The high-altitude polar NMs SOPO/SOPB have the atmospheric cut-off at $299 \mathrm{MeV}$ (physical isotropic) and $328 \mathrm{MeV}$ (instrumental), while the corresponding values for DOMC/DOMB are $283 \mathrm{MeV}$ and $314 \mathrm{MeV}$. Therefore, the cut-off energies of those stations are about $100 \mathrm{MeV}$ lower than ones of polar NMs at sea level.

This is important for classification of SEP events and clarification of the recently updated and established definitions of GLEs and sub-GLEs. The sub-class called sub-GLEs is defined as (a) a count rate increase in high-altitude polar NMs with low atmospheric cut-off, which is not seen at the sea level (Poluianov et al., 2017, [1]); and (b) as large SEP event with protons having energies $>300 \mathrm{MeV}$, but not intensive enough to be detected by ground-based NMs (Raukunen et al., 2018, [2]). Since the atmospheric cut-off energy of the high-altitude polar NMs is about $300 \mathrm{MeV}$ (Table 1), we conclude that these two definitions of sub-GLEs are in good agreement with each other. 


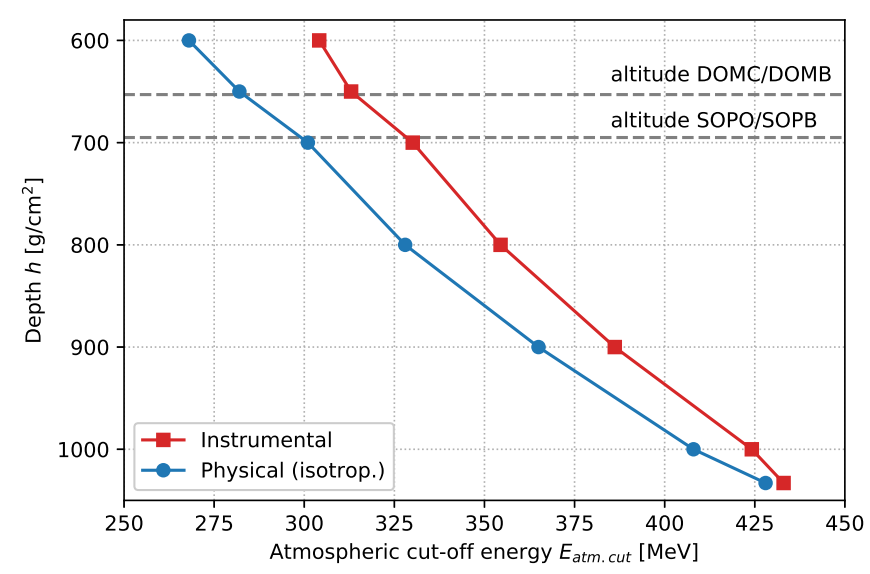

Figure 4: Atmospheric cut-off energy computed with the physical (isotropic incidence) and instrumental approaches. The horizontal dashed lines indicate the depths (altitudes) of the high-altitude polar neutron monitors DOMC/DOMB and SOPO/SOPB.

Table 1: Atmospheric cut-off energy $[\mathrm{MeV}]$ estimated with the physical and instrumental approaches.

\begin{tabular}{lccccc}
\hline \multirow{2}{*}{ Depth $\left[\mathrm{g} / \mathrm{cm}^{2}\right]$} & \multicolumn{2}{c}{ Physical } & \multicolumn{3}{c}{ Instrumental } \\
& Isotrop. CR & Vertical CR & $\phi=652 \mathrm{MV}$ & $300 \mathrm{MV}$ & $1200 \mathrm{MV}$ \\
\hline 600 & $268 \pm 3$ & $186 \pm 3$ & 304.15 & 302.41 & 305.33 \\
700 & $301 \pm 5$ & $225 \pm 4$ & 330.10 & 326.76 & 332.52 \\
800 & $328 \pm 6$ & $268 \pm 5$ & 354.47 & 352.56 & 355.83 \\
900 & $365 \pm 7$ & $302 \pm 7$ & 386.16 & 385.18 & 386.83 \\
1000 & $408 \pm 8$ & $342 \pm 8$ & 424.18 & 423.86 & 424.40 \\
1033 & $428 \pm 9$ & $357 \pm 8$ & 433.00 & 433.00 & 433.00 \\
\hline
\end{tabular}

\section{Acknowledgments}

We acknowledge the support from the Academy of Finland (projects 330064 QUASARE and 321882 ESPERA). The work was motivated by discussions in the International Space Science Institute International Team 441: High EneRgy sOlar partICle Events Analysis (HEROIC).

\section{References}

[1] S. Poluianov, I. Usoskin, A. Mishev, M. Shea and D. Smart, Gle and sub-gle redefinition in the light of high-altitude polar neutron monitors, Solar Physics 292 (2017) 176.

[2] O. Raukunen, R. Vainio, A.J. Tylka, W.F. Dietrich, P. Jiggens et al., Two solar proton fluence models based on ground level enhancement observations, Journal of Space Weather and Space Climate 8 (2018) A04. 
[3] S. Agostinelli, J. Allison and K. Amako, Geant4 - a simulation toolkit, Nuclear Instruments and Methods in Physics Research Section A: Accelerators, Spectrometers, Detectors and Associated Equipment 506 (2003) 250.

[4] L. Desorgher, E. Flückiger, M. Gurtner, M. Moser and R. Bütikofer, A geant 4 code for computing the interaction of cosmic rays with the earth's atmosphere, International Journal of Modern Physics A 20 (2005) 6802.

[5] J. Picone, A. Hedin, D. Drob and A. Aikin, Nrlmsise-00 empirical model of the atmosphere: Statistical comparisons and scientific issues, Journal of Geophysical Research: Space Physics 107 (2002) 1468.

[6] H. Carmichael, Cosmic rays (instruments), in Ann. IQSY, C.M. Minnis, ed., vol. 1, (Cambridge, MA), pp. 178-197, MIT Press, 1968.

[7] J. Clem and L. Dorman, Neutron monitor response functions, Space Sci. Rev. 93 (2000) 335.

[8] A. Mishev, I. Usoskin and G. Kovaltsov, Neutron monitor yield function: New improved computations, Journal of Geophysical Research 118 (2013) 2783.

[9] A.L. Mishev, S.A. Koldobskiy, G.A. Kovaltsov, A. Gil and I.G. Usoskin, Updated Neutron-Monitor Yield Function: Bridging Between In Situ and Ground-Based Cosmic Ray Measurements, J. Geophys. Res. Space Phys. 125 (2020) e27433.

[10] S.A. Koldobskiy, V. Bindi, C. Corti, G.A. Kovaltsov and I.G. Usoskin, Validation of the Neutron Monitor Yield Function Using Data from AMS-02 Experiment 2011 - 2017, J. Geophys. Res. (Space Phys.) 124 (2019) 2367.

[11] A. Gil, I. Usoskin, G. Kovaltsov, A. Mishev, C. Corti and V. Bindi, Can we properly model the neutronmonitor count rate?, J. Geophys. Res. 120 (2015) 7172.

[12] W. Nuntiyakul, A. Sáiz, D. Ruffolo, P.-S. Mangeard, P. Evenson, J. Bieber et al., Bare neutron counter and neutron monitor response to cosmic rays during a 1995 latitude survey, Journal of Geophysical Research: Space Physics 123 (2018) 7181.

[13] I.G. Usoskin, G.A. Kovaltsov, O. Adriani, G.C. Barbarino, G.A. Bazilevskaya et al., Force-field parameterization of the galactic cosmic ray spectrum: Validation for Forbush decreases, Adv. Space Res. 55 (2015) 2940.

[14] E. Vos and M. Potgieter, New modeling of galactic proton modulation during the minimum of solar cycle 23/24, Astrophysical Journal 815 (2015) 119.

[15] G. Villoresi, L. Dorman, N. Iucci and N. Ptitsyna, Cosmic ray survey to antarctica and coupling functions for neutron component near solar minimum (1996-1997): 1. methodology and data quality assurance, J. Geophys. Res. Space Phys. 105 (2000) 21025.

[16] W. Nuntiyakul, P. Evenson, D. Ruffolo, A. Sáiz, J.W. Bieber, J. Clem et al., Latitude Survey Investigation of Galactic Cosmic Ray Solar Modulation during 1994-2007, Astrophys. J. 795 (2014) 11. 